\title{
Visual quality assessment of the liver graft by the transplanting surgeon predicts postreperfusion syndrome after liver transplantation: a retrospective cohort study
}

Felix Kork ${ }^{1 *}$ D, Alexandra Rimek', Anne Andert ${ }^{2}$, Niklas Jurek Becker², Christoph Heidenhain ${ }^{3}$, Ulf P. Neumann², Daniela Kroy ${ }^{4}$, Anna B. Roehl ${ }^{1}$, Rolf Rossaint ${ }^{1}$ and Marc Hein ${ }^{1}$

\begin{abstract}
Background: The discrepancy between demand and supply for liver transplants (LT) has led to an increased transplantation of organs from extended criteria donors (ECD).

Methods: In this single center retrospective analysis of 122 cadaveric LT recipients, we investigated predictors of postreperfusion syndrome (PRS) including transplant liver quality categorized by both histological assessment of steatosis and subjective visual assessment by the transplanting surgeon using multivariable regression analysis. Furthermore, we describe the relevance of PRS during the intraoperative and postoperative course of LT recipients.

Results: $53.3 \%(n=65)$ of the patients suffered from PRS. Risk factors for PRS were visually assessed organ quality of the liver grafts (acceptable: OR 12.2 [95\% Cl 2.43-61.59], $P=0.002$; poor: OR 13.4 [95\% Cl 1.48-121.1], $P=0.02$ ) as well as intraoperative norepinephrine dosage before reperfusion (OR 2.2 [95\% Cl 1.26-3.86] per $0.1 \mathrm{\mu g} \mathrm{kg}^{-1} \mathrm{~min}^{-1}, P=0.01$ ). In contrast, histological assessment of the graft was not associated with PRS. LT recipients suffering from PRS were hemodynamically more instable after reperfusion compared to recipients not suffering from PRS. They had lower mean arterial pressures until the end of surgery $(P<0.001)$, received more epinephrine and norepinephrine before reperfusion ( $P=0.02$ and $P<0.001$, respectively) as well as higher rates of continuous infusion of norepinephrine $(P<0.001)$ and vasopressin $(P=0.02)$ after reperfusion. Postoperative peak AST was significantly higher $(P=0.001)$ in LT recipients with PRS. LT recipients with intraoperative PRS had more postoperative adverse cardiac events $(P=0.05)$ and suffered more often from postoperative delirium $(P=0.04)$.
\end{abstract}

Conclusions: Patients receiving ECD liver grafts are especially prone to PRS. Anesthesiologists should keep these newly described risk factors in mind when preparing for reperfusion in patients receiving high-risk organs.

Keywords: Steatosis, Hyponatremia, Cold ischemia time

\footnotetext{
* Correspondence: fkork@ukaachen.de

'Department of Anaesthesiology, Medical Faculty, RWTH Aachen University,

Pauwelsstrasse 30, 52074 Aachen, Germany

Full list of author information is available at the end of the article
} 


\section{Background}

According to the Organ Procurement and Transplantation Network of the United States (US) Department of Health and Human Services, the numbers of liver transplantations (LT) in the US have constantly been rising since the beginning of LT [1]: In 2016, a total of 7841 LTs were performed in the US. By contrast, the numbers of LTs in Germany and in the Eurotransplant region have been decreasing since a peak in 2010 [2, 3]. The decreasing organ donations combined with the persisting high morbidity and mortality of patients on the waiting list has led to a discrepancy between organ supply and demand $[2,4]$ and therefore to the more frequent acceptance extended criteria donors (ECD) to the pool of eligible donors $[5,6]$.

The most critical intraoperative moment for the anesthesiologist during LT is the reperfusion of the liver graft. An immediate and severe complication following reperfusion is hemodynamic instability, the so called postreperfusion syndrome (PRS). PRS is defined as a decrease of mean arterial pressure (MAP) of more than $30 \%$ during the first $5 \mathrm{~min}$ after reperfusion and continuing for at least 1 min [7-12]. PRS occurs in around 10-60\% of LT recipients $[8,9,13]$. Several risk factors for PRS have been described: Older donor age, higher donor risk index, longer cold ischemic time (CIT), severity of the recipient's liver disease, operation time and technique, hemodynamics at time of reperfusion, and steatosis of the graft organ [8, 13-20]. The mechanisms of PRS appear to be complex and not fully understood $[10,21,22]$. In addition, the transplantation of ECD livers has led to a decrease in mortality for $\mathrm{LT}$ recipients on the wait lists at the cost of increased perioperative complication [23]. Both these factors have made it difficult to predict PRS. In particular, the quality of the donor organ and its role as risk factor for the occurrence of PRS as well as its associations with patients' outcome have been neglected in the past.

\section{Methods}

\section{Aim, design and setting of the study}

We therefore conducted a retrospective analysis of all LTs performed at our center. Primary aim of this study was to identify predictors of PRS including transplant liver quality assessed by both subjective visual assessment by the transplanting surgeon and histological assessment of steatosis. Secondary aim of this study was to describe the relevance of PRS during the intraoperative and postoperative course of LT recipients.

\section{Patients and management}

All patients receiving cadaveric liver transplantation from the beginning of our center's newly established liver transplantation program in May 2010 until January 1st, 2014 were considered eligible for inclusion. Patients were excluded in case histological donor data or intraoperative data were incomplete or in case of intraoperative severe adverse events prior to reperfusion. Liver transplantation was performed using an extracorporeal venovenous/portal venous bypass. Anesthesiological management, bypass and surgical procedures, as well as the immune suppression regimen have already been described by Moosdorf and colleagues [24]. Anesthesiologists did not follow a specific coagulation or transfusion management protocol: Patient received at maximum 1 Liter of balanced electrolyte solution and volume replacement was subsequently conducted with FFP in order to anticipate the coagulation disorder. Transfusion triggers for RBCs were tailored to the patient's comorbidities and provided at the discretion of the providing anesthesiologist. Our department's standard operating procedure (SOP) for LT includes a TEM after induction, 15-30 and 45-60 min after reperfusion, seeking to keep normal coagulation parameters [25].

\section{Data \\ Donor data}

The following data were abstracted from the covering letter of the donor organ: donor age, donor body mass index, donor blood sodium concentration, donor alanine transaminase (ALT) blood concentration, donor aspartate transaminase (AST) blood concentration, donor bilirubin blood concentration, as well as warm ischemia time (WIT) and cold ischemia time (CIT).

\section{Donor organ assessment}

Donor livers were macroscopically assessed regarding their fat content by the implanting surgeon before the recipient operation on the preserved cold graft. Organs were categorized as either good, acceptable or poor, according to EuroTransplant criteria. In addition, histological assessment of the donor organ was conducted by the explanting center (or if missing by the in-house pathology department). Organs were categorized in three categories depending on macrovesicular fat content ( $\geq$ one intracellular vacuole displacing organelles): Grade 1 - fat content $0-29 \%$, grade 2 - fat content $30-59 \%$, grade 3 fat content $\geq 60 \%[26,27]$.

\section{Recipient data}

The following data were abstracted from the patient's medical chart, as recorded at the time of evaluation for being listed for transplantation: Recipient age, recipient diagnosis leading to transplantation, portal hypertension (PoHT; defined by either esophageal varices, thrombopenia or splenomegaly), laboratory model of end stage liver disease score (labMELD; 10 x (0,957 x In(serum creatinine $)+0,37 \times \operatorname{In}$ (serum creatinine $)+1,12 \times$ In (international normalized ratio[INR]) $+0,643)$ ) [28], and the 
need for renal replacement therapy. From the electronic patient data management system, concentrations of serum bilirubin, serum AST, serum ALT, serum creatinine, international normalized ratio (INR) as well as renal replacement therapy were abstracted after admission closest to the beginning of surgery (preoperatively), at ICU admission immediately after surgery (postoperatively) and on postoperative day (POD) 1,3,7 and 14 .

The following data were abstracted from the paper-based anesthesia protocol: Heart rate, mean blood pressure (arterial line), boli of norepinephrine and epinephrine at time of reperfusion, infusion rate of norepinephrine, infusion rate of epinephrine, infusion rate of vasopressin. These values were abstracted at induction of anesthesia, skin incision, beginning of the anhepatic phase, and at 5, 10, 15, 30 and $60 \mathrm{~min}$ after reperfusion as well as at the end of the surgery. Furthermore, the number of intraoperatively administered red blood cell concentrates (RBCs), platelet concentrates (PCs), fresh frozen plasmas (FFPs), the amount of intraoperatively administered fibrinogen, prothrombin complex concentrate (PCC), the occurrence of hyperfibrinolysis (by thrombelastometry [TEM]), asystole and cerebral edema (temporary mydriasis after reperfusion) were extracted from the anesthesia protocol.

From the patients chart we abstracted the following data from the postoperative period: primary nonfunction (PND; re-transplantation or death within 7 days), early allograft dysfunction (bilirubin $\geq 10 \mathrm{mg} / \mathrm{dl}$ on post-operative day (POD) 7 and/or INR $\geq 1.6$ on POD 7 and/or AST or ALT $>2000 \mathrm{IU} / \mathrm{L}$ within the first 7 days), acute rejection (clinical diagnosis), surgical revisions, retransplantation, sepsis, need for renal replacement therapy (RRT), adverse cardiovascular events (asystole, resuscitation, non ST elevation myocardial infarction, heart failure), adverse central nervous events (delirium, intracranial hemorrhage, seizures), duration of mechanical ventilation, intensive care unit (ICU) length of stay (LOS), hospital LOS, and death.

\section{Postreperfusion syndrome}

PRS was defined as fulfillment of at least one of the following criteria: (1) Decrease in mean arterial pressure (MAP) of at least $30 \%$ at time of reperfusion, (2) administration of an intravenous bolus of norepinephrine $>2 \mu \mathrm{g} \mathrm{kg}$ body weight $(\mathrm{BW})^{-1}$, (3) increase of continuous norepinephrine infusion of $\geq 0.1 \mu \mathrm{g} \mathrm{kg} \mathrm{BW}^{-1}$ within 5 to $30 \mathrm{~min}$ after reperfusion, or (4) initiation of continuous vasopressin infusion after reperfusion. According to our department's SOP, PRS was treated as follows: (i) $0.5 \mathrm{mg}$ atropine before reperfusion if heart rate $<80$, (ii) NE boli and NE infusion to maintain MAP, (iii) epinephrine boli and infusion in case of significant bradycardia with hypotension and decrease of $\mathrm{SVO}_{2}$ during reperfusion, (iv) infusion of vasopressin if high doses of NA are necessary or NA therapy ineffective.

\section{Statistics}

Categorical data with two categories each were tested using Fisher's exact test, with more than three categories with the Chi squared test with Yates correction. Since the sample size was $>100$, central limit theorem applies and normal distribution of continuous variables was assumed without testing. Groupwise comparison of continuous variables were therefore conducted using the $t$-test for independent samples. Changes of continuous variables over time were compared using a repeated measures ANOVA test. If a time/variable-interaction was detected by the ANOVA, a post-hoc $t$-test was conducted for each time point. Survival analysis were conducted plotting KaplanMeier curves and these were compared using the Log Rank test. Agreement between visual organ assessment by the surgeon and histopathological steatosis grading was quantified by calculating an unweighted Cohen's kappa. Binary logistic regression analysis was used to determine independent factors predicting postreperfusion syndrome. Variables reaching a level of significance $(P \leq 0.05)$ when univariably tested, were introduced into the multivariable logistic regression model. Statistical analyses were conducted using IBM SPSS 22, figures were created using GraphPad Prism 6.0. A two-sided $p$-value $\leq 0.05$ was considered statistically significant.

\section{Results \\ Patients}

A total of 172 patients received a liver transplantation during the study period. 50 patients were excluded due to incomplete histological data $(n=46)$, incomplete intraoperative documentation $(n=3)$ or due to severe adverse events prior to reperfusion ( $n=1$; need for cardiopulmonary bypass due to laceration of the inferior caval vein). A total of 122 patients were analyzed (Fig. 1).

Clinical characteristics of the study population are depicted in Table 1. Recipients of LT had a mean age of $55(54.9 \pm 9.8)$ and a mean labMELD of $20(19.8 \pm 10.3)$. The most frequent reason for transplantation was cirrhosis ( 57 of 122 ; $46.7 \%$ ) followed by tumor ( 25 of 122 ; $20.5 \%)$. Organ donors had a mean age of $55(55.2 \pm 16.2)$, were $46.7 \%$ female, had a mean BMI of $29(28.8 \pm 7.2)$, and serum sodium, ALT, AST, bilirubin within the reference range. The mean CIT of the donor organs was $8 \mathrm{~h}$ $(8.3 \pm 2.3)$, mean WIT was $44 \mathrm{~min}(43.7 \pm 7.5)$.

\section{Postreperfusion syndrome}

Of the 122 liver transplant recipients, 65 (53.3\%) developed PRS (Table 1). Patients developing PRS received an organ more often due to acute liver failure ( 10 of $65 \mathrm{vs.}$ 2 of $57,15.4 \%$ vs. $3.5 \%, P=0.03)$ and less often due to cirrhosis (20 of 65 vs. 37 of $57,30.8 \%$ vs. $64.9 \%, P<$ $0.001)$ compared to recipients not developing PRS and suffered more often from PoHT (52 of 65 vs. 33 of 57, 


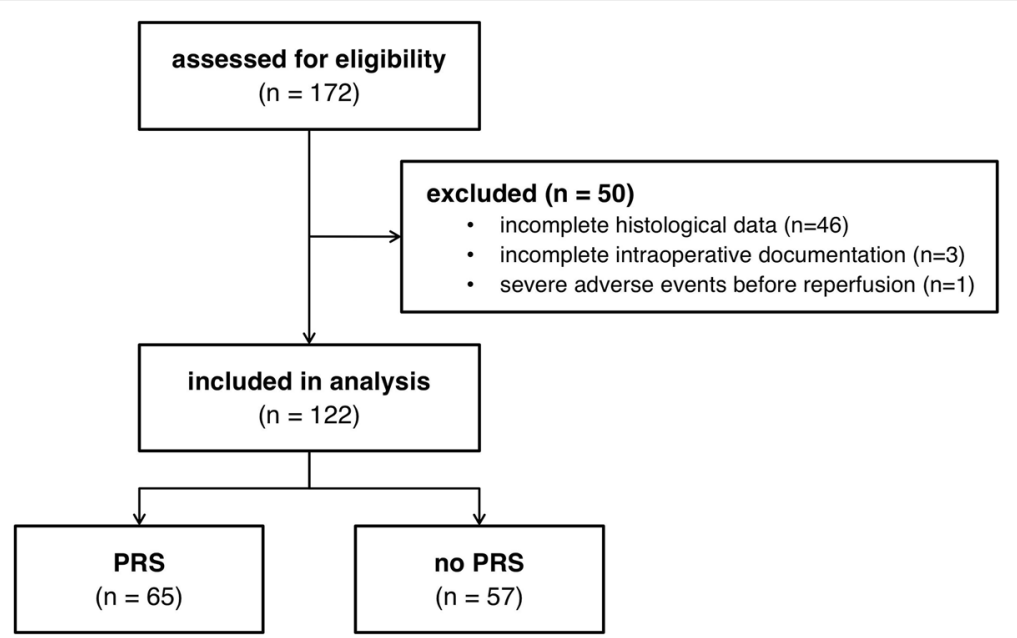

Fig. 1 Patients. Flow chart of patient inclusion. PRS: postreperfusion syndrome

$80.0 \%$ vs. $57.9 \%, P=0.01$ ). Organ donors for recipients developing PRS had a higher BMI $(31.1 \pm 8.2$ vs. $26.4 \pm$ $4.8, P<0.001)$ and a higher serum sodium concentration (149.1 \pm 7.8 vs. $146.1 \pm 8.4, p=0.04)$ compared to organ donors for recipients not developing PRS. Donor organs for patients who developed PRS had longer mean CITs $(8.7 \pm 2.3$ vs. $7.8 \pm 2.3 \mathrm{~h}, p=0.04)$ and more often had an extreme CIT of $\geq 10$ h (17 of 65 vs. 6 of $57,26.2 \%$ vs. $10.5 \%, p=0.02$ ).

\section{Predictors of postreperfusion syndrome}

In order to identify predictors of PRS we conducted a binary logistic regression analysis with PRS as dependent variable introducing known risk factors for PRS in the model (Table 2). Only visually assessed acceptable (OR 12.2 [95\% CI 2.43-61.59], $P=0.002$ ) or poor (OR 13.4 [95\% CI 1.48-121.1], $P=0.02$ ) quality of the donor organ and norepinephrine dosage before reperfusion (OR 2.2 [95\% CI 1.26-3.86] per $0.1 \mu \mathrm{g} \mathrm{kg}^{-1} \mathrm{~min}^{-1}, P=$ $0.01)$ predicted the occurrence of PRS. Interestingly, the histological assessment of donor organ's steatosis was not a good predictor for PRS. This was not due to collinearity of the variables: the visual and histological assessment differed significantly $(p<0.001)$ and agreed poorly (cohen's kappa 0.31, Table 3). For example, 21 of 122 donor organs were staged as acceptable or poor by visual inspection but classified as stage 1 steatosis by histological assessment.

\section{Postreperfusion syndrome and the intraoperative course after reperfusion}

LT recipients with PRS were hemodynamically more unstable after reperfusion until the end of surgery compared to patients without PRS (Fig. 2): Recipients with PRS had lower MAPs after reperfusion $(P<0.001$; Fig. $2 \mathrm{a})$ and accordingly received higher infusion of continuous norepinephrine $(P<0.001$, Fig. $2 \mathrm{~b})$ and vasopressin $(P<$ 0.001; Fig. 2c) compared to patients without PRS. Heart rate and continuous epinephrine infusion after reperfusion did not differ in LT recipients with and without PRS (Fig. 2d and e). At the time of reperfusion of the liver transplant, recipients with PRS received higher boli of norepinephrine $\left(1.5 \pm 1.6\right.$ vs. $0.3 \pm 0.4 \mu \mathrm{g} \mathrm{kg} \mathrm{kg}^{-1}, P=<$ $0.001)$ and epinephrine $\left(0.3 \pm 0.5\right.$ vs. $0.1 \pm 0.2 \mu \mathrm{g} \mathrm{kg}^{-1} ; P=$ 0.01; Fig. 2f).

Furthermore, LT recipients with PRS received more extensive hemotherapy intraoperatively: Compared to patients without PRS, LT recipients with PRS received more platelet concentrates $(1.6 \pm 1.8$ vs. $1.1 \pm 1.5$ units; $P=0.04$; more fibrinogen $(3.0 \pm 3.2$ vs. $1.7 \pm 1.2$ g; $P=$ $0.01)$ and more PCC $(1313 \pm 1610$ vs. $579 \pm 1133$ IU; $P=$ 0.01; Table 4). There was no significant difference in the amount of RBC and FFP administered as well as in other intraoperative postreperfusion adverse events (Table 4).

\section{Postreperfusion syndrome and the postoperative course after transplantation}

LT recipients with PRS demonstrated a more severe organ damage after transplantation: During the first three postoperative days, AST was higher in patients with PRS $(P=0.02$; Fig. 3a), as was ALT, but without statistical significance $(P=0.40$; Fig. $3 \mathrm{~b})$. LT recipients with and without PRS did not differ in bile retention, as postoperative serum bilirubin concentrations were similar (Fig. 3c). Recipients with PRS postoperatively had poorer organ function as hemostasis was poorer compared to recipients without PRS ( $P=0.01$; Fig. $3 d)$. The inflammation marker procalcitonin as well as the kidney function marker serum creatinine did not differ in LT recipients with and without PRS (Fig. 3e and f).

Regarding postoperative adverse events, LT recipients with PRS suffered more often from postoperative delirium 
Table 1 Clinical characteristics. of liver organ donors (top) and liver transplant recipients (bottom) of 122 single center liver transplantations (LTs)

\begin{tabular}{|c|c|c|c|c|}
\hline & All LTs $(n=122)$ & PRS $(n=65)$ & no PRS $(n=57)$ & $P$ \\
\hline \multicolumn{5}{|l|}{ Donor characteristics } \\
\hline Age [years] & $55.2 \pm 16.2$ & $57.1 \pm 15.1$ & $53.1 \pm 17.2$ & 0.18 \\
\hline Sex [female] & $57(46.7 \%)$ & $32(49.2 \%)$ & $24(42.1 \%)$ & 0.42 \\
\hline BMI $\left[\mathrm{kg} / \mathrm{cm}^{2}\right]$ & $28.8 \pm 7.2$ & $31.1 \pm 8.2$ & $26.4 \pm 4.8$ & $<0.001$ \\
\hline Sodium $[\mathrm{mmol} / \mathrm{L}]$ & $147.7 \pm 8.2$ & $149.1 \pm 7.8$ & $146.1 \pm 8.4$ & 0.04 \\
\hline $\mathrm{ALT}[\mathrm{U} / \mathrm{L}]$ & $114.2 \pm 289.6$ & $106.7 \pm 223.2$ & $122.8 \pm 352.4$ & 0.77 \\
\hline AST [U/L] & $124.4 \pm 237.8$ & $115.9 \pm 182.1$ & $134.1 \pm 290.0$ & 0.69 \\
\hline Bilirubine $[\mathrm{mg} / \mathrm{dL}]$ & $0.86 \pm 1.23$ & $0.80 \pm 1.33$ & $0.93 \pm 1.13$ & 0.59 \\
\hline Cold ischemic time $[\mathrm{h}]$ & $8.3 \pm 2.3$ & $8.7 \pm 2.3$ & $7.8 \pm 2.3$ & 0.04 \\
\hline$\geq 10 \mathrm{~h}[\mathrm{n}]$ & $23(18.9 \%)$ & $17(26.2 \%)$ & $6(10.5 \%)$ & 0.02 \\
\hline Warm ischemic time [min] & $43.7 \pm 7.5$ & $44.9 \pm 6.7$ & $42.3 \pm 8.2$ & 0.06 \\
\hline \multicolumn{5}{|l|}{ Recipient characteristics } \\
\hline Age [years] & $54.9 \pm 9.8$ & $55.2 \pm 9.8$ & $54.5 \pm 9.8$ & 0.69 \\
\hline Sex [female] & $43(35.2 \%)$ & $21(32.3 \%)$ & $22(38.6 \%)$ & 0.57 \\
\hline BMI $\left[\mathrm{kg} / \mathrm{cm}^{2}\right]$ & $27.6 \pm 5.3$ & $27.3 \pm 5.5$ & $28.1 \pm 5.0$ & 0.41 \\
\hline \multicolumn{5}{|l|}{ Diagnosis [n] } \\
\hline acute liver failure & $12(9.8 \%)$ & $10(15.4 \%)$ & $2(3.5 \%)$ & 0.03 \\
\hline acute on chronic & $5(4.1 \%)$ & $1(1.5 \%)$ & $4(7.0 \%)$ & 0.18 \\
\hline cirrhosis & $57(46.7 \%)$ & $20(30.8 \%)$ & 37 (64.9\%) & $<0.001$ \\
\hline tumor & $25(20.5 \%)$ & $14(21.5 \%)$ & $11(19.3 \%)$ & 0.82 \\
\hline graft failure & $7(5.7 \%)$ & $2(3.1 \%)$ & $5(8.8 \%)$ & 0.25 \\
\hline others (PSC, polycystic) & $16(13.1 \%)$ & $10(15.4 \%)$ & $6(10.5 \%)$ & 0.59 \\
\hline portal hypertension & $85(69.7 \%)$ & $52(80.0 \%)$ & $33(57.9 \%)$ & 0.01 \\
\hline labMELD & $19.8 \pm 10.3$ & $20.0 \pm 9.9$ & $19.6 \pm 10.8$ & 0.82 \\
\hline Creatinine preop $[\mathrm{mg} / \mathrm{dl}]$ & $1.71 \pm 1.49$ & $1.75 \pm 0.23$ & $1.66 \pm 0.15$ & 0.14 \\
\hline preoperative RRT [n] & $15(12.3 \%)$ & $8(12.3 \%)$ & $7(12.3 \%)$ & 1.00 \\
\hline
\end{tabular}

$L T$ Liver transplantation, PRS postreperfusion syndrome, BMI body mass index, ALT Alanine transferase, AST Aspartat transferase, labMELD laboratory Model of endstage liver disease score, $R R T$ renal replacement therapy

Clinical characteristics of liver organ donors (top) and liver transplant recipients (bottom) of 122 single center LTs. The characteristics are displayed for the whole study population (left) and by occurrence of postoperative reperfusion syndrome; mean and standard deviation (SD) or frequencies

( $8 / 65$ vs. $1 / 57,12.3 \%$ vs. $1.8 \% ; P=0.04)$ and major cardiovascular events $(11 / 65$ vs. $3 / 57,16.9 \%$ vs. $5.3 \% ; P=0.05$; Table 5). LT recipients with PRS also tended to have a longer ICU LOS $(11.7 \pm 17.0$ vs. $9.7 \pm 15.7 ; P=0.09$; Table 5$)$. A Kaplan-Meier survival analysis for LT recipient survival (Fig. 4a) and graft survival (Fig. 4b) did not show significant differences.

\section{Discussion}

In this single center retrospective analysis of 122 LT recipients, we found that approximately half of the patients suffered from PRS (53.3\%). Multivariable regression analysis identified two predictors of PRS: Only visually assessed acceptable (OR 12.2, $P=0.002$ ) and poor (OR 13.4, $P=0.02$ ) donor organ quality by the transplanting surgeon and intraoperative norepinephrine infusion rate before reperfusion of the liver transplant (OR 2.2 per $0.1 \mu \mathrm{g} / \mathrm{kg} / \mathrm{min}, p=$ 0.01) were associated with the occurrence of PRS. LT recipients suffering from PRS were hemodynamically more instable after reperfusion compared to recipients not suffering from PRS. They had lower MAPs from reperfusion until the end of surgery $(P<0.001)$. Hence, they received more epinephrine and norepinephrine before reperfusion $(P<0.001$ and $P=0.01$, respectively) and higher rates of continuous infusion of norepinephrine $(P<0.001)$ and vasopressin $(P=0.02)$ after reperfusion of the transplant. Moreover, LT recipients with intraoperative PRS had a more complicated postoperative course compared to recipients without intraoperative PRS: They had more adverse cardiac events $(P=$ 
Table 2 Predictors of PRS

\begin{tabular}{|c|c|c|c|c|c|c|c|c|}
\hline & \multicolumn{4}{|c|}{ univariable } & \multicolumn{4}{|c|}{ multivariable } \\
\hline & Wald & OR & $95 \% \mathrm{Cl}$ & $P$ & Wald & OR & $95 \% \mathrm{Cl}$ & $P$ \\
\hline \multicolumn{9}{|l|}{ Donor related } \\
\hline Age [years] & 1.79 & 1.02 & $0.99-1.04$ & 0.18 & & & & \\
\hline Sodium $[\mathrm{mmol} / \mathrm{L}]$ & 3.92 & 1.05 & $1.00-1.10$ & 0.048 & 3.74 & 1.06 & $1.0-1.13$ & 0.053 \\
\hline $\mathrm{ALT}[\mathrm{U} / \mathrm{L}]$ & 0.94 & 1.00 & $0.99-1.00$ & 0.76 & & & & \\
\hline AST [U/L] & 0.17 & 1.00 & $0.99-1.00$ & 0.68 & & & & \\
\hline Bilirubine [mg/dL] & 0.28 & 0.92 & $0.68-1.24$ & 0.60 & & & & \\
\hline CIT [h] & 4.09 & 1.19 & $1.05-1.41$ & 0.04 & 1.62 & 1.14 & $0.93-1.38$ & 0.20 \\
\hline WIT [min] & 3.54 & 1.05 & $0.99-1.11$ & 0.06 & & & & \\
\hline \multicolumn{9}{|l|}{ visual assessment by surgeon ${ }^{a}$} \\
\hline acceptable & 11.03 & 13.14 & $2.87-60.03$ & 0.001 & 9.21 & 12.23 & $2.43-61.59$ & 0.002 \\
\hline poor & 6.27 & 14.6 & $1.79-118.9$ & 0.01 & 5.34 & 13.40 & $1.48-121.1$ & 0.02 \\
\hline \multicolumn{9}{|l|}{ steatosis (histological) ${ }^{\mathrm{b}}$} \\
\hline stage 2 & 0.36 & 1.58 & $0.36-6.93$ & 0.55 & & & & \\
\hline stage 3 & 1.38 & 3.79 & $0.41-34.97$ & 0.42 & & & & \\
\hline \multicolumn{9}{|l|}{ Recepient related } \\
\hline labMELD & 0.05 & 1.00 & $0.97-1.04$ & 0.82 & & & & \\
\hline POHT & 6.78 & 2.91 & $1.30-6.50$ & $<0.01$ & 2.15 & 2.07 & $0.78-5.48$ & 0.14 \\
\hline NE dosage before reperfusion $\left[0.1 \mathrm{\mu g} \mathrm{kg}^{-1} \mathrm{~min}^{-1}\right]$ & 7.86 & 2.00 & $1.23-3.24$ & 0.01 & 7.56 & 2.20 & $1.26-3.86$ & 0.01 \\
\hline
\end{tabular}

ALT alanine transferase, AST aspartate transferase, CIT cold ischemia time, WIT warm ischemia time, labMELD laboratory Model of end-stage liver disease score, POHT portal hypertension, $N E$ norepinephrine, $O R$ odds ratio, $C l$ confidence interval

acompared to visual assessment category "good"; 'compared to stage 1

Results of univariable (left) and multivariable (right) binary logistic regression analyses in 122 cases of LT to predict PRS. Only visually assessed steatosis by the surgeon prior to implantation and NE dosage before reperfusion remained significant predictors of PRS in the multivariable model

$0.05)$ and suffered more often from postoperative delirium $(P=0.04)$.

All 122 LT recipients were operated at the same center with the same technique (venovenous/portalvenous bypass) and with uniform anesthesiologic management, guided by a SOP that included instructions for the management of PRS. No changes in operating technique or anesthesiologic management were made during the study period, leading to a homogenous single

Table 3 Differences in assessment of the graft organ between transplanting surgeon and histological examination in 122 cases of LTS

\begin{tabular}{lllll}
\hline $\begin{array}{l}\text { Transplanting } \\
\text { surgeons' } \\
\text { macroscopic } \\
\text { assessment }\end{array}$ & $\begin{array}{l}\text { Histological assessment of the macrovesical fat } \\
\text { content }^{\mathrm{a}}\end{array}$ & Total \\
\cline { 2 - 4 } & Grade 1 & Grade 2 & Grade 3 & \\
\hline Good & 88 & 2 & 1 & 91 \\
Acceptable & 17 & 3 & 0 & 20 \\
Poor & 4 & 3 & 4 & 11 \\
Total & 109 & 8 & 5 & 122 \\
\hline
\end{tabular}

${ }^{a}$ Grade 1: fat content $0-29 \%$, grade 2: fat content $30-59 \%$, grade 3: fat content $\geq 60 \%$

Agreement between these two methods of assessment was poor (cohen's kappa 0.31 ) and transplanting surgeons classified 21 organs as only acceptable or poor that were classified as grade 1 steatosis by histological assessment center study sample. Nevertheless, this study has several weaknesses. Due to the retrospective design of the analyses, data quality could be impaired. Histological data for our analyses was limited to steatosis assessment. Analyses of hemodynamics from hand-written anesthesia protocols are often suspected to present "sugar-coated" hemodynamic values. Although we cannot rule out that this was the case, our data present clinically and empirically plausible hemodynamics. At worst, hemodynamic stability is overestimated and the effects shown are even more pronounced. The fact that this was a single center analysis combined with the uncommon but consistent use of intraoperative venovenous/portalvenous bypass limits the external validity of our results.

Although rather on the top end of the range, PRS incidence of $53 \%$ in our sample concurs with several other studies $[8,10,12,17,19]$. The pathophysiology of PRS is complex and not entirely understood. The abrupt influx of cold, hyperkalemic and acidic blood into the circulation, air or thrombotic embolization and the release of vasoactive substances from the graft liver contribute to PRS [29-31]. The rationale for using a venovenous/portalvenous bypass during the anhepatic phase at our center is to ensure maximum safety of the procedure [24]. The combination of a femoro-brachial and porto-axillary 


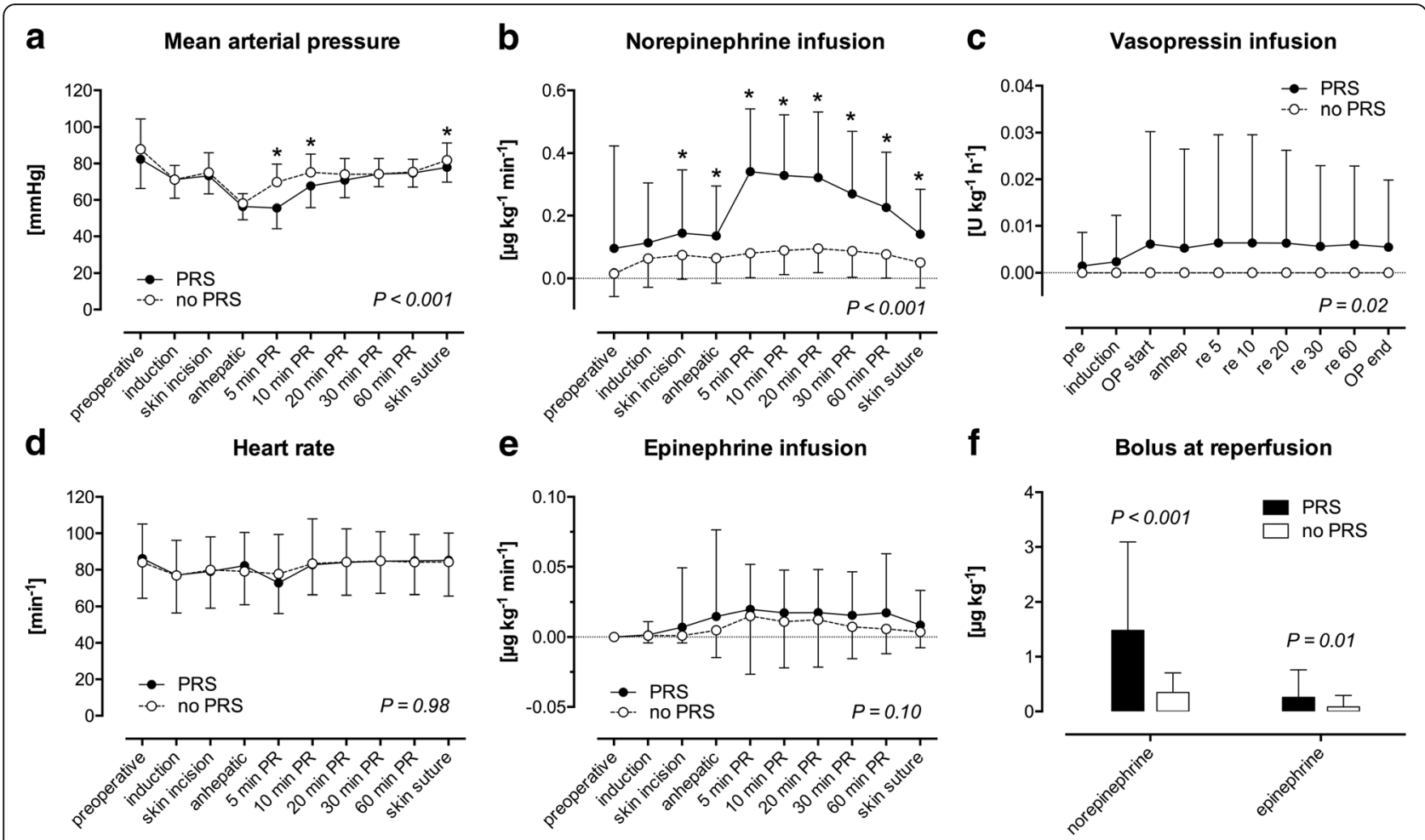

Fig. 2 Hemodynamics. Intraoperative hemodynamics of 122 liver transplant (LT) recipients, 65 with postreperfusion syndrome (PRS, black circles), 57 without postoperative PRS (white circles). LT recipients with PRS were hemodynamically more unstable compared to patients without PRS. Mean arterial pressure after reperfusion was lower (a), accordingly, norepinephrine (b) and vasopressin infusion (c) were higher in LT recipients with PRS compared to recipients without PRS. Heart rate (d) and epinephrine infusion (e) did not differ. At the time of reperfusion, LT recipients with PRS received greater boli of norepinephrine and epinephrine (f). P-values: repeated measures ANOVA; *: post-hoc $t$-test; mean and standard deviation

bypass reduces lower limb and mesenterial congestion and therefore reduces the abrupt influx of hyperkalemic and acidic blood into systemic circulation at the time of reperfusion. Nevertheless, the use of a bypass during LT surgery remains controversial $[13,32,33]$. The PRS effect seen in our study is therefore likely to be predominantly

Table 4 Intraoperative adverse events

\begin{tabular}{llll}
\hline & PRS $(n=65)$ & no PRS $(n=57)$ & $P$ \\
\hline Hyperfibrinolysis $^{\mathrm{a}}[\mathrm{n}]$ & $2(3.1 \%)$ & $2(3.5 \%)$ & 1.00 \\
Asystole $[\mathrm{n}]$ & $0(0.0 \%)$ & $1(1.8 \%)$ & $\mathrm{n} / \mathrm{a}^{\mathrm{c}}$ \\
Cerebral edema $^{\mathrm{b}}[\mathrm{n}]$ & $0(0.0 \%)$ & $1(1.8 \%)$ & $\mathrm{n} / \mathrm{a}^{\mathrm{c}}$ \\
Transfusions & & & \\
RBC [U] & $10.7 \pm 7.3$ & $9.5 \pm 9.0$ & 0.12 \\
FFP [U] & $18.0 \pm 9.2$ & $17.2 \pm 10.3$ & 0.50 \\
Platelet concentrate [U] & $1.6 \pm 1.8$ & $1.1 \pm 1.5$ & 0.04 \\
Fibrinogen [g] & $3.0 \pm 3.2$ & $1.7 \pm 2.6$ & 0.01 \\
PCC [IU] & $1313 \pm 1610$ & $579 \pm 1133$ & 0.01 \\
\hline
\end{tabular}

PRS postreperfusion syndrome, $R B C$ red blood cell concentrate, FFP fresh frozen plasma, $P C C$ prothrombin complex concentrate

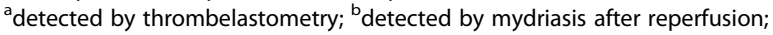

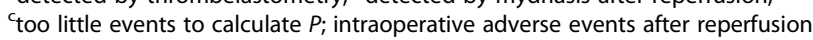
in 122 liver transplant recipients apportioned by patients with and without postreperfusion syndrome (PRS) caused by vasoactive substances released from the graft. With that in mind, PRS incidence does appear rather high.

A possible explanation for this contradiction may be the fact that we used an extended definition for PRS. PRS is commonly defined as a decrease in MAP of more than $30 \%$ from the baseline value for more than one minute during the first five minutes after reperfusion [7, 8, 10-12]. We extended the definition for PRS for mainly two reasons. First, the treatment of PRS seeks to preserve hemodynamic stability. At the time of reperfusion, anesthesiologists expect a certain degree of hemodynamic instability and preemptively treat a (soon to be) falling MAP with catecholamines [11], either as bolus or by increasing continuous infusion of the very same. Since this preemptive treatment conceals the occurrence of by-definition-PRS, we added (i) the administration of an intravenous bolus of norepinephrine $>2 \mu \mathrm{g} \mathrm{kg}(\mathrm{BW})^{-1}$ as well as (ii) the increase of continuous norepinephrine infusion of $\geq 0.1 \mu \mathrm{g} \mathrm{kg} \mathrm{BW}{ }^{-1}$ within the first $5 \mathrm{~min}$ after reperfusion as new criteria to the definition. Second, our clinical empiricism and the literature have presented cases of prolonged vasodilation after reperfusion [34-36]. We thus included (iii) an increase of continuous norepinephrine infusion up to $30 \mathrm{~min}$ after reperfusion and (iv) the initiation of continuous vasopressin 


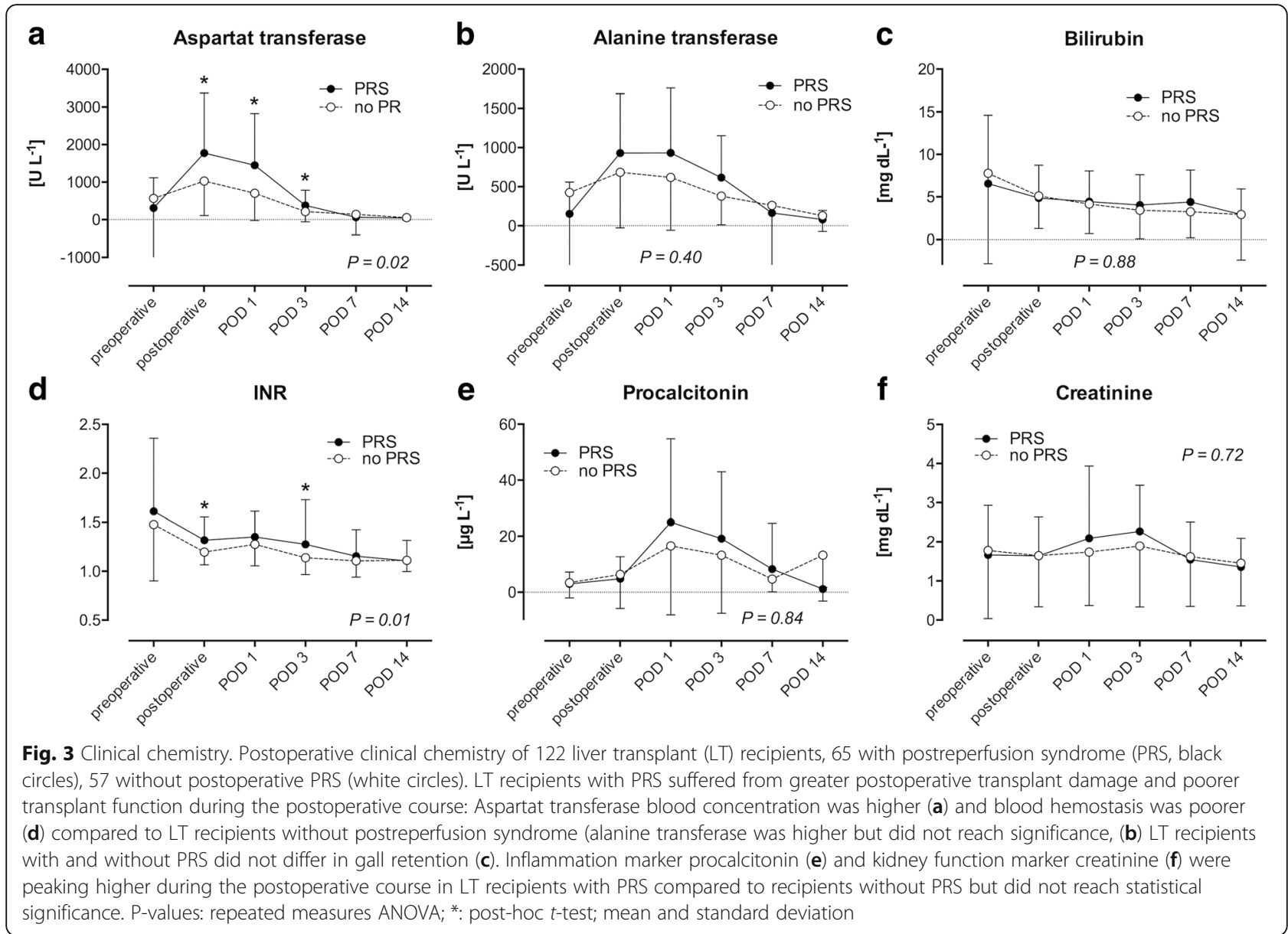

infusion after reperfusion as additional criteria for PRS. This altered definition impedes comparability with other studies and may overestimate PRS incidence compared to the commonly used definition. However, our data appear clinically plausible and concur with description of hemodynamic recovery after PRS by other authors [17].

In this study, multivariable regression modelling identified one donor related and one recipient related predictor of PRS: graft quality - visually assessed by the transplanting surgeon - and cumulative intraoperative norepinephrine dosage before reperfusion of the liver graft. The variety of risk factors and risk prediction models described in different studies suggests that PRS may occur in an unpredictable manner or may be highly subjective to center-specific effects $[8,13-20,22]$. The most frequent reported risk factors for PRS are longer CIT $[15,16,18,20]$ and intraoperative hemodynamics prior to reperfusion $[14,15,17,20]$, followed by graft steatosis $[8,16]$, operation time and technique $[15,18]$, severity of recipient's liver disease $[8,19]$, and donor age $[13,17]$. One single center study retrospective analysis was unable to identify even a single risk factor in a retrospective analysis of a sample of 261 LT recipients [37]. Our findings that graft quality and hemodymic impairment prior to reperfusion are risk factors for PRS concur with several similar studies. However, it remains cryptic why the multitude of these observational studies generate such a diversity of findings when it comes to predicting postreperfusion syndrome. Prospective multicenter trials, the creation of an LT register or meta-analyses investigating the prediction of PRS could help to shed light on this matter in the future.

The increasing number of liver transplantations has led to an increased demand of donor organs [1] and to the growing use of organs from extended criteria donors (ECD) [23]. A common quality marker for organs is fatty change, or steatosis. Steatosis has indeed been linked to the occurrence of PRS $[8,16]$. Studies have shown that transplantation of liver grafts with moderate to severe steatosis can very well be conducted without sacrificing recipient survival but in particular, that increased efforts are needed when dealing with these organs [26, 38]. However, macroscopic assessment of the organ may not be qualified to assess steatosis: Rey and colleagues have examined 36 livers of organ donors which were explanted but not allocated. 
Table 5 Postoperative outcome

\begin{tabular}{llll}
\hline & PRS $(n=65)$ & no PRS $(n=57)$ & $P$ \\
\hline Early allograft dysfunction [n] & $25(38.5 \%)$ & $16(28.1 \%)$ & 0.25 \\
Retransplantation [n] & $5(7.7 \%)$ & $4(7.0 \%)$ & 0.75 \\
$\quad$ Due to primary non function ${ }^{\mathrm{a}}$ [n] & $3(4.6 \%)$ & $1(1.8 \%)$ & \\
Due to thrombosis [n] & $0(0.0 \%)$ & $1(1.8 \%)$ & \\
Acute rejection [n] & $12(18.5 \%)$ & $13(22.8 \%)$ & 0.66 \\
Surgical revision [n] & $26(40.0 \%)$ & $25(43.9 \%)$ & 0.72 \\
$\quad$ Bleeding [n] & $22(33.8 \%)$ & $19(33.3 \%)$ & 1.00 \\
Severe infection/sepsis [n] & $11(16.9 \%)$ & $8(14.0 \%)$ & 0.80 \\
Renal function [n] & & & \\
RRT [n] & $27(41.5 \%)$ & $20(35.1 \%)$ & 0.27 \\
Major cardiovascular events ${ }^{\mathrm{b}}$ [n] & $11(16.9 \%)$ & $3(5.3 \%)$ & 0.05 \\
Adverse CNS events [n] & & & \\
Delirium [n] & $8(12.3 \%)$ & $1(1.8 \%)$ & 0.04 \\
Intracranial bleeding [n] & $3(4.6 \%)$ & $0(0.0 \%)$ & 0.25 \\
Seizures [n] & $1(1.5 \%)$ & $1(1.8 \%)$ & 1.00 \\
Extubation in the OR [n] & $15(23.1 \%)$ & $20(35.1 \%)$ & 0.16 \\
ICU LOS [days] & $11.7 \pm 17.0$ & $9.7 \pm 15.7$ & 0.09 \\
Hospital LOS [days] & $36.5 \pm 18.2$ & $35.6 \pm 23.9$ & 0.24 \\
Deaths [n] & $10(15.4 \%)$ & $7(12.3 \%)$ & 0.33 \\
Due to sepsis/MOF [n] & $6(9.2 \%)$ & $6(10.5 \%)$ & \\
Due to cardiovascular events [n] & $2(3.1 \%)$ & $1(1.8 \%)$ & \\
Due to bleeding [n] & $1(1.5 \%)$ & $0(0.0 \%)$ & \\
Due to carcinoma [n] & $1(1.5 \%)$ & $0(0.0 \%)$ & \\
\hline
\end{tabular}

Short-term postoperative outcome of 122 liver transplant recipients with and without postreperfusion syndrome (PRS); RRT renal replacement therapy, CNS central nervous system, ICU intensive care unit; LOS: length of stay MOF multi organ failure $P R S$ postreperfusion syndrome

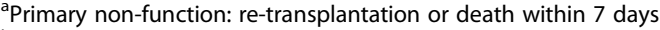

${ }^{\mathrm{b}}$ major cardiovascular events: asystole, resuscitation, non ST elevation myocardial infarction

The authors found that macroscopic appearance and coloring compared to histopathologic evaluation for steatosis unveiled discrepancies: Most of the graft livers with histopathologically confirmed mild degree of steatosis macroscopically appeared yellow, thus of false poor quality and could have been transplanted. In our analyses, microscopic steatosis evaluation was a worse predictor for PRS than the visual evaluation of the transplanting surgeon. This may be the case because surgeons likely assess the organ not only by its color, but also by general appearance, organ consistency, and size. It may even be the case that information of the recipient's medical history and the knowledge that the organ was already rejected once by another center or had a long CIT additionally influenced the assessment. Therefore, the multitude of information may have enabled the surgeon to predict PRS better than any single variable (recipient status, CIT, steatosis, etc.).

We also found intraoperative norepinephrine infusion rate before reperfusion to be a predictor of PRS. This also concurs with other studies that have identified markers of hemodynamic instability prior to reperfusion as predictors of PRS. One possible explanation for norepinephrine infusion or hemodynamic instability is hypovolemia. This would also concur with other studies that have shown lower central venous pressure at time of dissection [14] or at time of reperfusion [17] as well as a higher requirement for transfusion units [15] to be predictors of PRS. A second possible explanation is vasodilatation or vasplegia which has been linked to end stage liver disease [39, 40]. Case reports have described vasoplegic syndrome to occur during liver transplantation [31, 41]. However, differentiation between the two can sometimes be complicated as both appear alike [34]. A third possible explanation may be that these patients already had a compromised cardiac function. E.g., Xu and colleagues could link left ventricular diastolic dysfunction to the occurrence of PRS in a cohort of 330 Chinese LT recipients [20]. Unfortunately, our data did not contain systematic and detailed cardiac function assessment of the recipients and the literature lacks systematic investigation of its impact on the occurrence of PRS. Moreover, it has been suggested by several authors that PRS could be only a sign of an underlying problem. It could be that anhepatic hypovolemia, vasoplegia and/or impaired cardiac function demask as PRS intraoperatively. On top of it, patients receiving a LT from ECD appear especially susceptible to severe PRS.
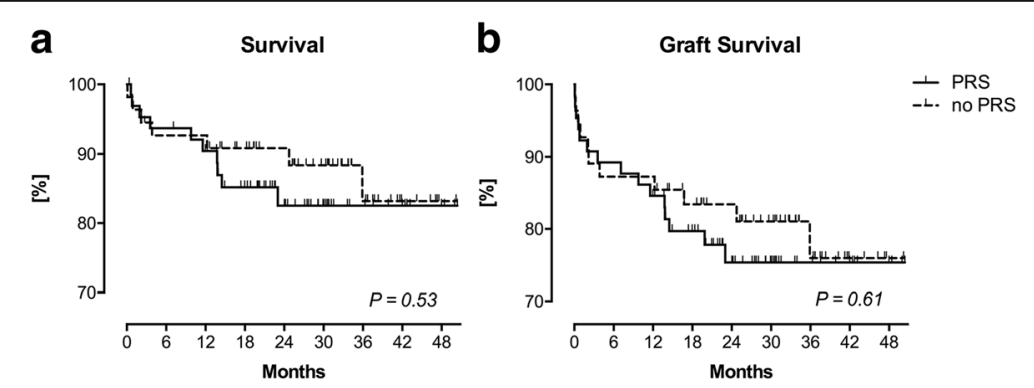

Fig. 4 Survival. Kaplan-Meier survival analysis of 122 liver transplant (LT) recipients, 65 with postreperfusion syndrome (PRS; solid line) and 57 with PRS (broken line). Survival analyses were conducted for LT recipient survival (a) as well as graft survival (retransplantation or recipient death; (b) PRS: postreperfusion syndrome 
In this light, these patients should especially be optimized regarding hemodynamics and acid-base balance when awaiting reperfusion.

Similar to the prediction of PRS, data on the effect of PRS on LT recipients' outcome is inconclusive. In particular, the impact of PRS on hard clinical endpoints such as primary graft nonfunction with requirement for retransplantation and mortality is unresolved. E.g., some studies show an effect of PRS on mortality [15, 18-20] and others did not $[13,17]$. In fact, we were not able to show a significant association of PRS with primary nonfunction (4.6 vs. $1.8 \%)$ and mortality ( $15.4 \%$ vs. $12.3 \%)$ in this study population. This is most likely due to the small sample size, again underscoring the need for multicenter registries. Regarding post-transplantation morbidity, we could demonstrate in our sample that the occurrence of PRS was associated with a higher frequency of postoperative delirium $(12.3 \%$ vs. $1.8 \%)$ and major cardiovascular events $(16.9 \%$ vs. $5.3 \%)$. While other studies have demonstrated early allograft dysfunction [42, 43], ICU length of stay [43] and the need for postoperative renal dysfunction $[19,20]$ we could not substantiate these associations in our study sample.

\section{Conclusions}

The data of this study demonstrated that both the visual assessment of the liver graft by the transplanting surgeon as well as the intraoperative recipients need for catecholamines before reperfusion of the graft were predictors for PRS at our center. Besides the known risk factors for PRS, anesthesiologists should keep these newly described risk factors in mind when preparing for reperfusion. As the impact of PRS on short- and long-term hard clinical endpoint remains inconclusive, further investigations and possibly multicenter prospective registries could ultimately resolve the impact of PRS on LT recipients' outcome.

\begin{abstract}
Abbreviations
ALT: Alanine transferase; AST: Aspartat transferase; CIT: Cold ischemia time; ECD: Extended criteria donors; FFP: Fresh frozen plasma; INR: International normalized ratio; labMELD: Laboratory Model of end stage liver disease; LT: Liver transplantation; MAP: Mean arterial pressure; MELD: Model of end stage liver disease; min: Minute(s); NE: Norepinephrine; OR: Odds ratio; PC: Platelet concentrate; PCC: Prothrombin complex concentrate; POD: Postoperative day; PoHT: Portal hypertension; PRS: Postreperfusion syndrome; PSC: Primary biliary cirrhosis; RBC: Red blood cell concentrate; SOP: Standard operating procedure; TEM: Thrombelastometry; US: United States; WIT: Warm ischemia time
\end{abstract}

\section{Acknowledgements}

This analysis was conducted in the course of the doctoral thesis of AR at the Medical Faculty of the RWTH Aachen, Germany.

\section{Funding}

The study did not receive external funding and was conducted on departmental funds only.

\section{Availability of data and materials}

The datasets generated and/or analysed during the current study are not publicly available due German Data Protection Laws but are available from the corresponding author on reasonable request after approval of the local ethics committee and data safety board.

\section{Authors' contributions}

$A R, A A, M H$ designed the research, AR participated in acquisition of the data, FK, AR, MH conducted data analysis. FK drafted the manuscript, AR and $\mathrm{MH}$ participated in writing of the manuscript, all authors interpreted the data and revised the manuscript critically for important intellectual content, approved the final version to be published and agreed to be accountable for all aspects of the work.

\section{Ethics approval and consent to participate}

The local ethics committee (University Hospital RWTH Aachen, EK 291/13) approved the analysis and waived the requirement of informed consent.

\section{Consent for publication}

Not applicable.

\section{Competing interests}

The authors declare that they have no competing interests.

\section{Publisher's Note}

Springer Nature remains neutral with regard to jurisdictional claims in published maps and institutional affiliations.

\section{Author details}

${ }^{1}$ Department of Anaesthesiology, Medical Faculty, RWTH Aachen University, Pauwelsstrasse 30, 52074 Aachen, Germany. ${ }^{2}$ Department of General, Visceral and Transplantation Surgery, Medical Faculty, RWTH Aachen University, Pauwelsstrasse 30, 52074 Aachen, Germany. ${ }^{3}$ Department of General, Visceral and Oncological Surgery, Sana Hospital Gerresheim, Gräulinger Strasse 120, 40625 Düsseldorf, Germany. ${ }^{4}$ Department of Medicine III, Medical Faculty, RWTH Aachen University, Pauwelsstrasse 30, 52074 Aachen, Germany.

Received: 15 January 2018 Accepted: 2 March 2018

Published online: 09 March 2018

\section{References}

1. Tranplants by Donor Type [https://optn.transplant.hrsa.gov/data/view-datareports/national-data/]. Accessed 10 May 2017.

2. Tacke F, Kroy DC, Barreiros AP, Neumann UP. Liver transplantation in Germany. Liver Transpl. 2016;22(8):1136-42.

3. Annual Report 2015 [http://www.eurotransplant.org/cms/mediaobject. php?file=AR_ET_20153.pdf]. Accessed 8 May 2017.

4. Schlitt HJ, Loss M, Scherer MN, Becker T, Jauch KW, Nashan B, Schmidt H, Settmacher $U$, Rogiers $X$, Neuhaus $P$, et al. Current developments in liver transplantation in Germany: MELD-based organ allocation and incentives for transplant centres. Z Gastroenterol. 2011;49(1):30-8.

5. Pezzati D, Ghinolfi D, De Simone P, Balzano E, Filipponi F. Strategies to optimize the use of marginal donors in liver transplantation. World J Hepatol. 2015;7(26):2636-47.

6. Routh D, Sharma S, Naidu CS, Rao PP, Sharma AK, Ranjan P. Comparison of outcomes in ideal donor and extended criteria donor in deceased donor liver transplant: a prospective study. Int J Surg. 2014;12(8):774-7.

7. Aggarwal S, Kang Y, Freeman JA, Fortunato FL, Pinsky MR. Postreperfusion syndrome: cardiovascular collapse following hepatic reperfusion during liver transplantation. Transplant Proc. 1987;19(4 Suppl 3):54-5.

8. Chung IS, Kim HY, Shin YH, Ko JS, Gwak MS, Sim WS, Kim GS, Lee S-K. Incidence and predictors of post-reperfusion syndrome in living donor liver transplantation. Clin Transpl. 2012;26(4):539-43.

9. Hilmi I, Horton CN, Planinsic RM, Sakai T, Nicolau-Raducu R, Damian D, Gligor S, Marcos A. The impact of postreperfusion syndrome on short-term patient and liver allograft outcome in patients undergoing orthotopic liver transplantation. Liver Transpl. 2008;14(4):504-8.

10. Lee J, Yoo YJ, Lee JM, Park YJ, Ryu HG. Sevoflurane versus Desflurane on the incidence of Postreperfusion syndrome during living donor liver transplantation: a randomized controlled trial. Transplantation. 2016;100(3):600-6. 
11. Ryu H-G, Jung C-W, Lee H-C, Cho Y-J. Epinephrine and phenylephrine pretreatments for preventing postreperfusion syndrome during adult liver transplantation. Liver Transpl. 2012;18(12):1430-9.

12. Ryu $H G$, Jung CW, Lee CS, Lee J. Nafamostat mesilate attenuates Postreperfusion syndrome during liver transplantation. Am J Transplant. 2011;11(5):977-83.

13. Nanashima A, Pillay P, Crawford M, Nakasuji M, Verran DJ, Painter D. Analysis of postrevascularization syndrome after orthotopic liver transplantation: the experience of an Australian liver transplantation center. J Hepato-BiliaryPancreat Surg. 2001;8(6):557-63.

14. Ayanoglu HO, Ulukaya S, Tokat Y. Causes of postreperfusion syndrome in living or cadaveric donor liver transplantations. Transplant Proc. 2003;35(4):1442-4.

15. Bukowicka B, Akar RA, Olszewska A, Smoter P, Krawczyk M. The occurrence of postreperfusion syndrome in orthotopic liver transplantation and its significance in terms of complications and short-term survival. Ann Transplant. 2011;16(2):26-30.

16. Chui AK, Shi L, Tanaka K, Rao AR, Wang LS, Bookallil M, Mayr M, Chiu E, Verran DJ, Mears D, et al. Postreperfusion syndrome in orthotopic liver transplantation. Transplant Proc. 2000;32(7):2116-7.

17. Fukazawa K, Yamada Y, Gologorsky E, Arheart KL, Pretto EAJ. Hemodynamic recovery following postreperfusion syndrome in liver transplantation. J Cardiothorac Vasc Anesth. 2014;28(4):1006-14.

18. Paugam-Burtz C, Kavafyan J, Merckx P, Dahmani S, Sommacale D, Ramsay M, Belghiti J, Mantz J. Postreperfusion syndrome during liver transplantation for cirrhosis: outcome and predictors. Liver Transpl. 2009;15(5):522-9.

19. Siniscalchi A, Dante A, Spedicato S, Riganello L, Zanoni A, Cimatti M, Pierucci E, Bernardi E, Miklosova Z, Moretti C, et al. Hyperdynamic circulation in acute liver failure: reperfusion syndrome and outcome following liver transplantation. Transplant Proc. 2010;42(4):1197-9.

20. Xu ZD, Xu HT, Yuan HB, Zhang H, Ji RH, Zou Z, Fu ZR, Shi XY. Postreperfusion syndrome during orthotopic liver transplantation: a singlecenter experience. Hepatobiliary Pancreat Dis Int. 2012:11(1):34-9.

21. DiNorcia J, Lee MK, Harlander-Locke MP, Xia V, Kaldas FM, Zarrinpar A, Farmer DG, Yersiz H, Hiatt JR, Busuttil RW, et al. Damage control as a strategy to manage Postreperfusion hemodynamic instability and coagulopathy in liver transplant. JAMA Surg. 2015;150(11):1066-72.

22. Jeong SM. Postreperfusion syndrome during liver transplantation. Korean Anesthesiol. 2015;68(6):527-39.

23. Pareja E, Cortes M, Hervas D, Mir J, Valdivieso A, Castell JV, Lahoz A. A score model for the continuous grading of early allograft dysfunction severity. Liver Transpl. 2015;21 (1):38-46.

24. Mossdorf A, Ulmer F, Junge K, Heidenhain C, Hein M, Temizel I, Neumann UP, Schoning W, Schmeding M. Bypass during liver transplantation: anachronism or revival? Liver transplantation using a combined Venovenous/portal venous bypass-experiences with 163 liver transplants in a newly established liver transplantation program. Gastroenterol Res Pract. 2015;2015:967951.

25. Gorlinger K. Coagulation management during liver transplantation. Hamostaseologie. 2006;26(3 Suppl 1):S64-76.

26. Angele MK, Rentsch M, Hartl WH, Wittmann B, Graeb C, Jauch KW, Loehe F. Effect of graft steatosis on liver function and organ survival after liver transplantation. Am J Surg. 2008;195(2):214-20.

27. Adam R, Reynes M, Johann M, Morino M, Astarcioglu I, Kafetzis I, Castaing D, Bismuth $\mathrm{H}$. The outcome of steatotic grafts in liver transplantation. Transplant Proc. 1991:23(1 Pt 2):1538-40.

28. Kamath PS, Wiesner RH, Malinchoc M, Kremers W, Therneau TM, Kosberg CL, D'Amico G, Dickson ER, Kim WR. A model to predict survival in patients with end-stage liver disease. Hepatology. 2001;33(2):464-70.

29. Zalunardo MP, Schlapfer M, Beck-Schimmer B, Seifert B, Spahn DR, Bettex D. Impact of cytokine release on ventricular function after hepatic reperfusion: a prospective observational echocardiographic study with tissue Doppler imaging. BMC Anesthesiol. 2015;15:107.

30. Nakasuji M, Bookallil MJ. Pathophysiological mechanisms of postrevascularization hyperkalemia in orthotopic liver transplantation. Anesth Analg. 2000;91 (6):1351-5.

31. Bezinover D, Kadry Z, Uemura T, Sharghi M, Mastro AM, Sosnoski DM, Dalal P, Janicki PK. Association between plasma cyclic guanosine monophosphate levels and hemodynamic instability during liver transplantation. Liver Transpl. 2013;19(2):191-8

32. Acosta F, Rodriguez MA, Sansano T, Contreras RF, Reche M, Roques V, Beltran R, Robles R, Bueno FS, Ramirez P, et al. Influence of surgical technique on postreperfusion syndrome during liver transplantation. Transplant Proc. 1999:31(6):2380-1.

33. Jugan E, Albaladejo $P$, Jayais $P$, Ecoffey $C$. The failure of venovenous bypass to prevent graft liver postreperfusion syndrome. Transplantation. 1992;54(1):81-4.

34. de Armas LC, Castillo YA: Is it possible to distinguish between vasoplegic syndrome and postreperfusion syndrome during liver graft reperfusion? Anesth Analg 2010, 110(3):969-970; author reply 970-961.

35. Cao Z, Gao Y, Tao G. Vasoplegic syndrome during liver transplantation. Anesth Analg. 2009;108(6):1941-3.

36. Bezinover D, Kadry Z, McCullough P, McQuillan PM, Uemura T, Welker K, Mastro AM, Janicki PK. Release of cytokines and hemodynamic instability during the reperfusion of a liver graft. Liver Transpl. 2011;17(3):324-30.

37. Kiseleva EA, Ushakova IA, Kim EF, Matveev GP, Biriulina N, Vabishchevich AV. To the issue of postreperfusion syndrome predictors in orthotopic liver transplantation (OLT). Anesteziol Reanimatol. 2012:2:31-5.

38. McCormack L, Petrowsky H, Jochum W, Mullhaupt B, Weber M, Clavien P-A. Use of severely steatotic grafts in liver transplantation: a matched casecontrol study. Ann Surg. 2007;246(6):940-6. discussion 946-948

39. Berzigotti A, Erice E, Gilabert R, Reverter E, Abraldes JG, Garcia-Pagan JC, Bosch J. Cardiovascular risk factors and systemic endothelial function in patients with cirrhosis. Am J Gastroenterol. 2013;108(1):75-82.

40. Wagener G, Kovalevskaya G, Minhaz M, Mattis F, Emond JC, Landry DW. Vasopressin deficiency and vasodilatory state in end-stage liver disease. J Cardiothorac Vasc Anesth. 2011;25(4):665-70.

41. Khosravi MB, Milani S, Ghaffaripour S, Sahmeddini A, Eghbal MH, MalekHosseini SA. Very high dose epinephrine for the treatment of Vasoplegic syndrome during liver transplantation. Int J Organ Transplant Med. 2013:4(1):32-4.

42. Reich DL, Wood RK Jr, Emre S, Bodian CA, Hossain S, Krol M, Feierman D. Association of intraoperative hypotension and pulmonary hypertension with adverse outcomes after orthotopic liver transplantation. J Cardiothorac Vasc Anesth. 2003;17(6):699-702.

43. Hilmi IA, Damian D, Al-Khafaji A, Sakai T, Donaldson J, Winger DG, Kellum JA. Acute kidney injury after orthotopic liver transplantation using living donor versus deceased donor grafts: a propensity score-matched analysis. Liver Transpl. 2015;21(9):1179-85.

\section{Submit your next manuscript to BioMed Central and we will help you at every step:}

- We accept pre-submission inquiries

- Our selector tool helps you to find the most relevant journal

- We provide round the clock customer support

- Convenient online submission

- Thorough peer review

- Inclusion in PubMed and all major indexing services

- Maximum visibility for your research

Submit your manuscript at www.biomedcentral.com/submit
C) Biomed Central 